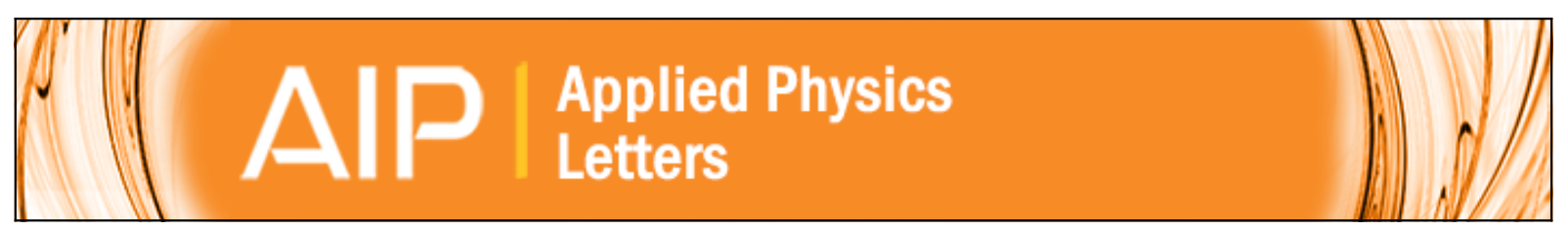

\title{
Efficient electron refrigeration using superconductor/spin-filter devices
}

Shiro Kawabata, Asier Ozaeta, Andrey S. Vasenko, Frank W. J. Hekking, and F. Sebastián Bergeret

Citation: Applied Physics Letters 103, 032602 (2013); doi: 10.1063/1.4813599

View online: http://dx.doi.org/10.1063/1.4813599

View Table of Contents: http://scitation.aip.org/content/aip/journal/apl/103/3?ver=pdfcov

Published by the AIP Publishing

\section{Articles you may be interested in}

Cooling of bulk material by electron-tunneling refrigerators

Appl. Phys. Lett. 86, 173508 (2005); 10.1063/1.1914966

Practical electron-tunneling refrigerator

Appl. Phys. Lett. 84, 625 (2004); 10.1063/1.1644326

Refrigerator with phonon filters: An application of the phonon deficit effect in superconducting tunnel junctions J. Appl. Phys. 94, 4619 (2003); 10.1063/1.1602946

Ultraefficient cooling in ferromagnet-superconductor microrefrigerators

Appl. Phys. Lett. 80, 3784 (2002); 10.1063/1.1481242

Integrated SINIS refrigerators for efficient cooling of cryogenic detectors

AIP Conf. Proc. 605, 375 (2002); 10.1063/1.1457666

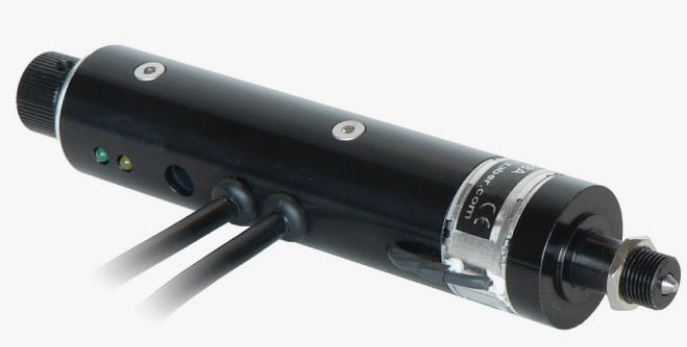

Automate your set-up with 


\title{
Efficient electron refrigeration using superconductor/spin-filter devices
}

\author{
Shiro Kawabata, ${ }^{1}$ Asier Ozaeta, ${ }^{2}$ Andrey S. Vasenko, ${ }^{3}$ Frank W. J. Hekking, ${ }^{3,4}$ \\ and F. Sebastián Bergeret ${ }^{2,5}$ \\ ${ }^{1}$ Electronics and Photonics Research Institute (ESPRIT), National Institute of Advanced Industrial Science \\ and Technology (AIST), Tsukuba, Ibaraki 305-8568, Japan \\ ${ }^{2}$ Centro de Física de Materiales (CFM-MPC), Centro Mixto CSIC-UPVIEHU, Manuel de Lardizabal 5 , \\ E-20018 San Sebastián, Spain \\ ${ }^{3}$ LPMMC, Université Joseph Fourier and CNRS, 25 Avenue des Martyrs, BP 166, 38042 Grenoble, France \\ ${ }^{4}$ Institut Universitaire de France, 103, bd Saint-Michel, 75005 Paris, France \\ ${ }^{5}$ Donostia International Physics Center (DIPC), Manuel de Lardizabal 4, E-20018 San Sebastián, Spain
}

(Received 22 May 2013; accepted 25 June 2013; published online 15 July 2013)

\begin{abstract}
Efficient electron-refrigeration based on a normal-metal/spin-filter/superconductor junction is proposed and demonstrated theoretically. The spin-filtering effect leads to values of the cooling power much higher than in conventional normal-metal/nonmagnetic-insulator/superconductor coolers and allows for an efficient extraction of heat from the normal metal. We demonstrate that highly efficient cooling can be realized in both ballistic and diffusive multi-channel junctions in which the reduction of the electron temperature from $300 \mathrm{mK}$ to around $50 \mathrm{mK}$ can be achieved. Our results indicate the practical usefulness of spin-filters for efficiently cooling detectors, sensors, and quantum devices. (C) 2013 AIP Publishing LLC. [http://dx.doi.org/10.1063/1.4813599]
\end{abstract}

The flow of charge current in N/I/S (normal metal/insulator/superconductor) tunnel junctions at a bias voltage $V$ is accompanied by a heat transfer from $\mathrm{N}$ into $\mathrm{S}$. This phenomenon arises due to the presence of the superconducting energy-gap $\Delta$ which allows for a selective tunneling of highenergy "hot" quasiparticles out of N. Such a heat transfer through N/I/S junctions can be used for the realization of microcoolers. ${ }^{1-4}$ Present state-of-the-art experiments allow the reduction of the electron temperature in a normal metal lead from 300 to about $100 \mathrm{mK}$, offering perspectives for onchip cooling of nano or micro systems, such as highsensitive sensors, detectors, and quantum devices. ${ }^{5-7}$

However, a serious limitation of the performance of N/I/ $\mathrm{S}$ microcoolers arises from the intrinsic multi-particle nature of charge transport in N/I/S junctions which is governed not only by single-particle tunneling but also by two-particle processes due to the Andreev reflection. ${ }^{3}$ While the singleparticle current and the associated heat current are due to quasiparticles with energies $E>\Delta$, at low temperatures or high junction transparencies the charge transport in N/I/S junctions is dominated by a subgap process: the Andreev reflection. In such a process, electrons with energies smaller than $\Delta$ are reflected as holes at the N/I/S interface, leading to the transfer of a Cooper pair into the superconductor. Since the energy of the electrons and holes involved in the process are symmetric with respect to the Fermi energy, there is no heat current through the interface. However, by applying a subgap bias across the junctions the Andreev reflection results in a finite charge current $I_{A}$ flowing through the N/I/S system. Due to finite resistance of the normal metal, this current generates Joule heating $I_{A} V$, which is entirely deposited in the normal metal. ${ }^{8-10}$ This heating exceeds the single-particle cooling at temperatures low enough, and therefore the suppression of Andreev processes is desirable for an efficient cooling.

One way to decrease the Andreev current is by decreasing the N/I/S junction transparency. However, large contact resistance hinders "hot" carrier transfer and leads to a severe limitation in the achievable cooling powers. In order to increase the junction transparency and at the same time to reduce the Andreev current, it was suggested to use ferromagnetic metals (FM). ${ }^{11,12}$ Giazotto and co-workers have theoretically investigated the cooling of a clean $\mathrm{N} / \mathrm{FM} / \mathrm{S}$ junction and predicted an enhancement of the cooling power compared to N/I/S junctions due to the suppression of the Andreev Joule heating. ${ }^{11}$ In order to realize such an efficient cooler, however, challenging half-metallic $\mathrm{FMs}^{13}$ with extremely high spin-polarization $P>0.94$ are needed.

In the present work we theoretically propose an alternative $\mathrm{N} / \mathrm{I} / \mathrm{S}$ microcooler with a ferromagnetic insulator as a tunneling barrier which acts as a spin-filter. This was demonstrated in experiments using europium chalcogenides tunneling barriers. ${ }^{14}$ The spin-filtering effect suppresses the Andreev reflection in a N/spin-filter(SF)/S junction as the one shown in Fig. 1(a). ${ }^{15,16}$ We show that this suppression leads, in both ballistic and diffusive N/SF/S junctions, to dramatic enhancement of the cooling power which gives rise to a dramatic reduction of the final achievable electron temperature.

In order to illustrate the basic cooling mechanism using spin-filters we first consider a one-dimensional clean N/SF/S junction [Fig. 1(a)]. In the following we set $\hbar=1$. The SF barrier can be modeled by a spin-dependent delta-function potential [see Fig. 1(a)], i.e., $V_{\sigma}(x)=\left(V+\rho_{\sigma} U\right) \delta(x)$, where $V$ is the spin-independent part of the potential, $U$ is the exchange-splitting, and $\rho_{\sigma}=-(+) 1$ for up (down) spins. ${ }^{15,17}$ The degree of the spin-filtering is characterized by the spinfiltering efficiency $P=\left|t_{\uparrow}-t_{\downarrow}\right| /\left(t_{\uparrow}+t_{\downarrow}\right)$, where $t_{\sigma}$ $=1 /\left[1+\left(Z+\rho_{\sigma} S\right)^{2}\right]$ is the transmission probability of the SF barrier for spin $\sigma$ with $m, k_{F}, Z \equiv m V / k_{F}$, and $S \equiv m U / k_{F}$ being the mass of electrons, the Fermi wave number, the normalized spin-independent, and -dependent potential 
(a)

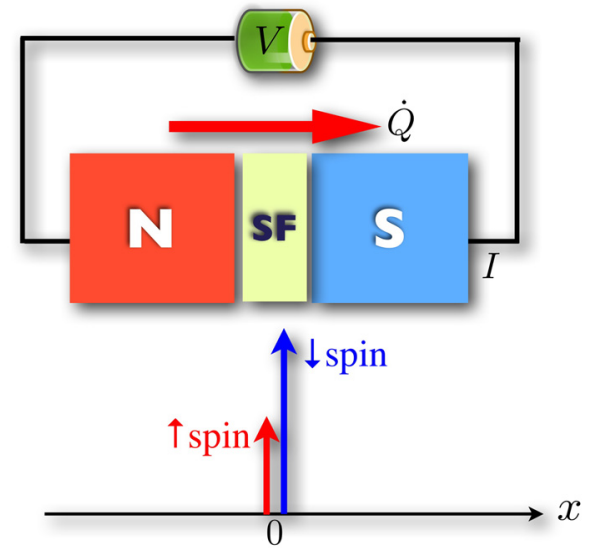

(b)

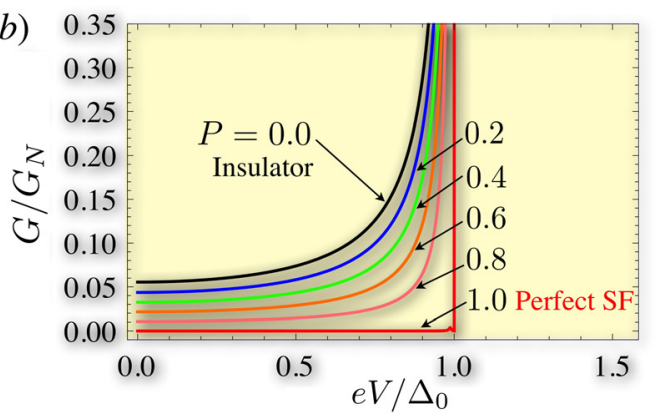

FIG. 1. (a) The schematic of a clean normal-metal/spin-filter/superconductor (N/SF/S) cooler and the delta-function model of an SF barrier which allows the spin-selective tunneling and the suppression of the Andreev reflection. (b) The differential conductance $G$ for various values of $P$ vs the bias voltage $V$ at $T=0 \mathrm{~K}$ for an N/SF/S junction with $t_{\uparrow}=0.1$. $G_{N}$ is the conductance of a N/SF/N junction, $\Delta_{0}$ is the superconducting gap at $T=0 \mathrm{~K}$, and $P$ is the spin-filtering efficiency.

barrier-height, respectively. For a perfect $\mathrm{SF}\left(t_{\uparrow}>0\right.$ and $t_{\downarrow}=0$ ), we get $P=1$.

The normal-reflection probability $B_{\sigma}$ and the Andreevreflection probability $A_{\sigma}$ of the junction are obtained by solving the Bogoliubov-de Gennes equation

$$
\left[\begin{array}{cc}
H_{0}-\rho_{\sigma} U \delta(x) & \Delta(x) \\
\Delta^{*}(x) & -H_{0}+\rho_{\sigma} U \delta(x)
\end{array}\right] \Phi_{\sigma}(x)=E \Phi_{\sigma}(x),
$$

together with the appropriate boundary conditions at the SF barrier $(x=0),{ }^{15}$ where $H_{0}$ is the spin-independent part of the single-particle Hamiltonian, i.e., $H_{0}=-\nabla^{2} / 2 m+V \delta(x)$ $-\mu_{F}, \Delta(x)=\Delta(T) e^{i \phi} \Theta(x)$ is a pair potential $[\phi$ is the phase of the pair potential and $\Theta(x)$ is the Heaviside step function], $\Phi_{\sigma}(x)$ is the eigenfunction, and the eigenenergy $E$ is measured from the chemical potential $\mu_{F}$.

We first focus on the spin-dependent charge-transport of the junction and address the suppression of the Andreev reflection by the spin-filtering effect. The voltage $V$ dependence of the differential conductance $G$ of the system can be calculated from the Blonder-Tinkham-Klapwijk formula, ${ }^{18}$ $G=\left.\left(e^{2} / 2 \pi\right) \sum_{\sigma=\uparrow . \downarrow}\left(1-B_{\sigma}+A_{\sigma}\right)\right|_{E=e V}$. In Fig. 1(b) we plot the spin-filtering efficiency $P$ dependence of $G / G_{N}$ vs $\mathrm{eV} / \Delta_{0}$ for a junction with $t_{\uparrow}=0.1$ at $T=0 \mathrm{~K}$, where $G_{N}$ $=\left(e^{2} / 2 \pi\right)\left(t_{\uparrow}+t_{\downarrow}\right)$ is the conductance of a N/SF/N junction and $\Delta_{0} \equiv \Delta(T=0 \mathrm{~K})$. When $P$ is increased, the sub-gap conductance for $|e V| \leq \Delta_{0}$ is largely reduced. ${ }^{15}$ Importantly if $P=1$, the Andreev reflection is completely inhibited, indicating that the SF would suppress the unwanted Andreev Joule heating.

To see the benefit of the spin-filtering effect on the electron cooling, we calculate the cooling power (the amount of heat extracted from $\mathrm{N}$ to $\mathrm{S}$ ) by using the Bardas and Averin formula, ${ }^{8} \dot{Q}=(e / \pi) \sum_{\sigma=\uparrow, \downarrow} \int_{-\infty}^{\infty} d E\left[E\left(1-B_{\sigma}-A_{\sigma}\right)\right.$ $\left.-e V\left(1-B_{\sigma}+A_{\sigma}\right)\right][f(E-e V)-f(E)]$, where $f(E)$ is the Fermi-Dirac distribution-function. The positive (negative) $\dot{Q}$ means the cooling (heating) of $\mathrm{N}$. In the calculation, we have determined $\Delta(T)$ by solving the BCS gap equation numerically. The $P$ dependence of the cooling power $\dot{Q}$ vs the bias voltage $V$ for $t_{\uparrow}=0.1$ is shown in Fig. 2(a). We have assumed that $T=0.5 T_{c}$, where $T_{c}$ is the superconducting transition temperature. If we increase $P$, the cooling power $\dot{Q}$ is largely enhanced. This result is attributed to the suppression of the Andreev reflection and the undesirable Andreev Joule heating. Therefore we can conclude that the spinfiltering effect boosts dramatically the cooling power with respect to conventional N/I/S coolers. In Fig. 2(b) we plot the spin-filtering efficiency $P$ dependence of the cooling-power $\dot{Q}_{\max }$ at $T=0.5 T_{c}$ and the optimal bias voltage $V=V_{\text {opt }}$ in which $\dot{Q}$ is maximized as a function of $V$. The maximum cooling-power $\dot{Q}_{\max }$ can be achieved in the case of the perfect SF $(P=1)$ because of the complete suppression of the Andreev reflection. Notably for the case of a large $t_{\uparrow}=0.3$ and $P=1$, the amount of heat extracted from $\mathrm{N}$ can be about a factor of 15 larger than the theoretical upper-limit of $\dot{Q}_{\max }$ for conventional N/I/S coolers $\left[\dot{Q}_{\max } \approx 0.001\left(\Delta_{0}^{2} / 2 \pi\right)\right]$,
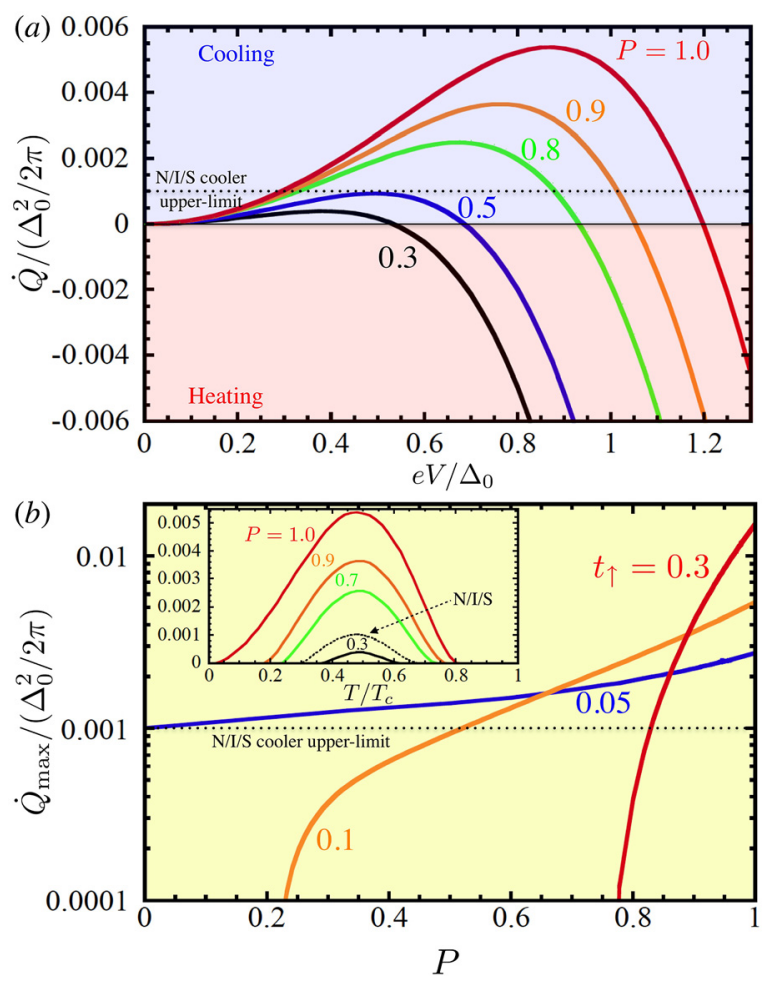

FIG. 2. (a) The cooling power $\dot{Q}$ vs the bias voltage $V$ of a clean N/SF/S refrigerator with $t_{\uparrow}=0.1$ at $T=0.5 T_{c}$ for several spin filtering efficiencies $P$. (b) The maximum cooling power $\dot{Q}_{\max }$ as a function of $P$ at $T=0.5 T_{c}$ for several values of $t_{\uparrow}$. The dotted line is the theoretical upper-limit of $\dot{Q}_{\max } \approx$ $0.001\left(\Delta_{0}^{2} / 2 \pi\right)$ for $\mathrm{N} / \mathrm{I} / \mathrm{S}$ refrigerators, which is achieved in the case of $T / T_{c} \approx 0.5$ and $t_{\uparrow}=t_{\downarrow} \approx 0.05$. Inset: The temperature $T$ dependence of $\dot{Q}_{\max }$ for several values of $P$. The dashed curve shows $\dot{Q}_{\max }$ for an N/I/S refrigerator with $t_{\uparrow}=t_{\downarrow}=0.05$. 
which can be achieved for $t_{\uparrow}=t_{\downarrow} \approx 0.05$ and $T / T_{c} \approx 0.5$ [see the dotted line in Fig. 2(b)]. ${ }^{8}$ It is crucial to note that even for small $P$ values [e.g., $P>0.0$ for $t_{\uparrow}=0.05$ ], $\dot{Q}_{\max }$ overcomes the upper limit of N/I/S coolers. By calculating the temperature dependence of $\dot{Q}_{\max }$, we also found that the $\dot{Q}_{\max }$ is maximized at around $T \approx 0.5 T_{c}$ irrespective of the value of $P$ [see the inset in Fig. 2(b)].

To discuss the practical applicability of SF-based coolers, next we consider a more realistic diffusive N/SF/S junction in which the elastic scattering length $l \ll \xi$, where $\xi=\sqrt{D / 2 \Delta}$ is the superconducting coherence length and $D$
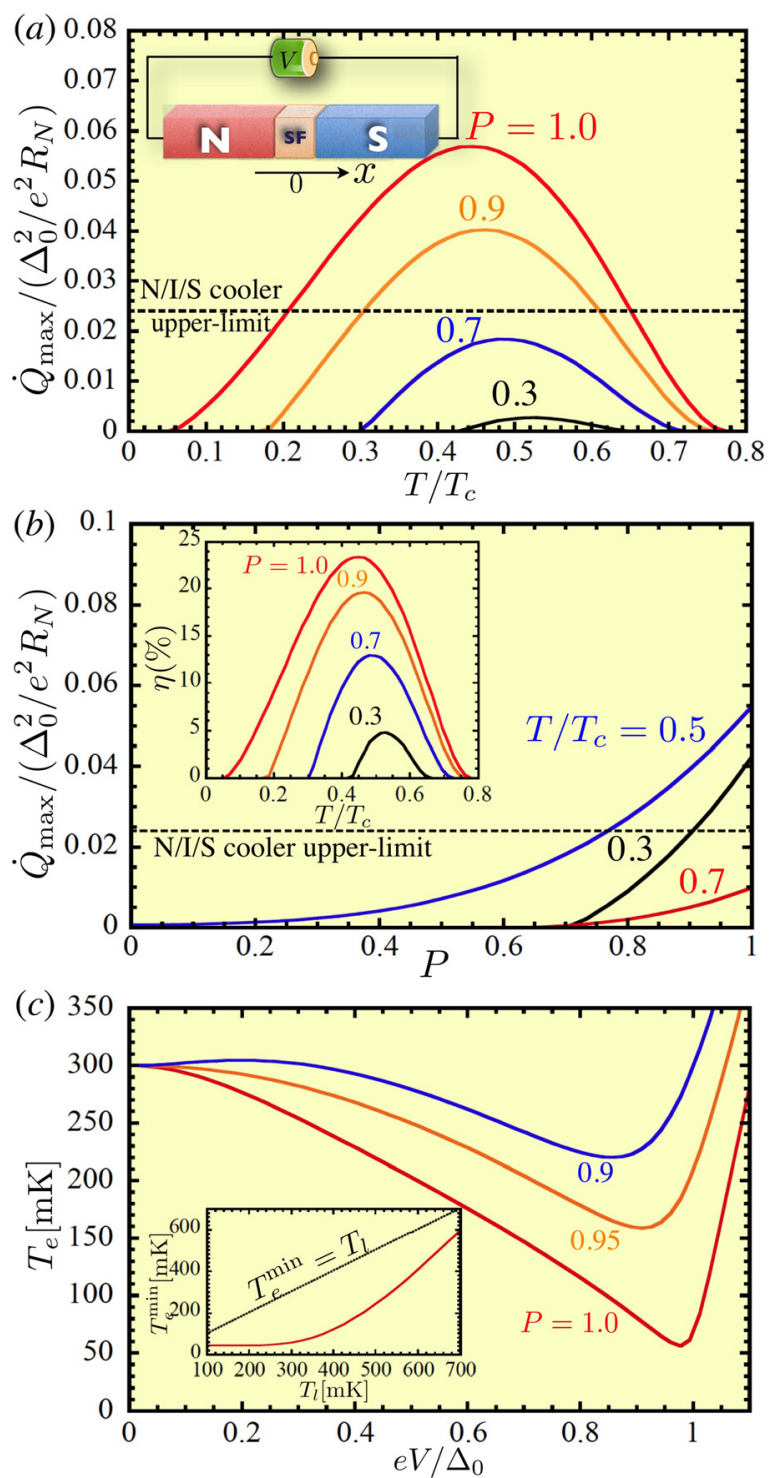

FIG. 3. (a) The maximum cooling power $\dot{Q}_{\max }$ as a function of the temperature $T$ for a diffusive N/SF/S junction shown in the inset and different values of $P$. (b) The dependence of $\dot{Q}_{\text {max }}$ on $P$ for $T / T_{c}=0.7$ (red), $T / T_{c}=0.5$ (blue), and $T / T_{c}=0.3$ (black). Other parameters are $\mathcal{A}=1 \mu \mathrm{m}^{2}$, $\sigma_{N}=0.015(\mu \Omega \mathrm{cm})^{-1}$, and $R_{N}=1.0 \mathrm{M} \Omega$. Inset: The refrigeration efficiency $\eta$ as a function of $T$. The horizontal dotted line in (a) and (b) is the theoretical upper-limit of $\dot{Q}_{\max }$ for diffusive N/I/S junctions, which is achieved in the case of $\mathcal{A}=1 \mu \mathrm{m}^{2}, \quad \sigma_{N}=0.015(\mu \Omega \mathrm{cm})^{-1}, \quad R_{N}=2.7 \mathrm{M} \Omega, \quad$ and $T / T_{c}=0.45$. (c) The electron temperature $T_{e}$ as a function of $V$ for different values of $P$ and $T_{l}=T_{S}=300 \mathrm{mK}$. Inset: the minimum electron temperature $T_{e}^{\min }$ as a function of the starting lattice temperature $T_{l}\left(=T_{e}\right.$ at $\left.V=0\right)$ for $P=1$. As a reference we show $T_{e}^{\min }=T_{l}$ line. We have chosen $\Sigma=2 \times 10^{-9} \mathrm{WK}^{-5} \mu \mathrm{m}^{-3}, \mathcal{V}=0.5 \mu \mathrm{m}^{3}$, and $\Delta=180 \mu \mathrm{eV}$. is the diffusion coefficient (in the following for simplicity we assume the same $D$ in the whole structure). We assume that the SF is a tunnel barrier and the $\mathrm{N}$ reservoir is infinite along the $x$ direction [see the inset in Fig. 3(a)].

The cooling power is given by the expression $\dot{Q}=-I^{E}-I V$, where $I$ and $I^{E}$ are the charge and energy currents, respectively. One can express $\dot{Q}$ in terms of the contributions from the single-particle $(|E|>\Delta)$ and the Andreev $(|E|<\Delta)$ processes ${ }^{10}$ as $\dot{Q}=\dot{Q}_{1}+\dot{Q}_{A}$, where $\dot{Q}_{1}=-I_{1}^{E}-I_{1} V$ and $\dot{Q}_{A}=-I_{A}^{E}-I_{A} V$. Here $I_{1(A)}^{E}$ and $I_{1(A)}$ are, respectively, the single-particle (Andreev) energy-current and the singleparticle (Andreev) charge-current. Importantly, the contribution of the Andreev processes to the energy current vanishes, $I_{A}^{E}=0$. Therefore the Andreev process contributes only to the Joule heating (i.e., $\dot{Q}_{A}=-I_{A} V$ ), which is fully released in the $\mathrm{N}$ electrode and leads to a severe reduction of the cooling power.

In order to compute the charge and energy currents through the junction we use the quasiclassical Green's function (GF) technique. These are given by ${ }^{19} I=\left(\sigma_{N} \mathcal{A} / 8 e\right)$ $\int_{0}^{\infty} d E \operatorname{Tr}\left[\tau_{3} \breve{J}^{K}\right]$ and $I^{E}=\left(\sigma_{N} \mathcal{A} / 8 e^{2}\right) \int_{0}^{\infty} d E E \operatorname{Tr}\left[\tau_{0} \breve{J}^{K}\right]$, where $\sigma_{N}$ is the conductivity of $\mathrm{N}, \mathcal{A}$ is the junction area, $\tau_{0}$ is the unit matrix, $\tau_{3}$ is the Pauli matrix in the Nambu space, $J$ $=\breve{G} \partial_{x} \breve{G}$ is the matrix current, and $\breve{G}$ is the quasiclassical GF which is a $8 \times 8$ matrix in the Keldysh $\times$ Nambu $\times$ spin space. In order to compute the currents $I$ and $I_{E}$ we assume a large SF barrier resistance $R_{N}$ such that $\xi /\left(R_{N} \sigma_{N} \mathcal{A}\right) \ll 1$. This assumption allows for a linearization of the Usadel equation $^{20}$ in the normal metal. ${ }^{12}$ The effect of the spin-filter barrier is included in the boundary conditions for the Usadel equation which has been recently derived in Ref. 16. According to the latter reference the Andreev reflection is proportional to $\sqrt{1-P}$. Thus, by increasing $P$ we expect a suppression of the unwanted Andreev Joule heating, i.e., an enhancement of the cooling power, as it turns our from our quantitative analysis below.

By following the procedure described above, we compute numerically the cooling power $\dot{Q}$ of our system as a function of the different parameters. We assume a junction area $\mathcal{A}=1 \mu \mathrm{m}^{2}$, a conductivity of $\mathrm{N} \sigma_{N}=0.015(\mu \Omega \mathrm{cm})^{-1}, 6$ and $R_{N}=1.0 \mathrm{M} \Omega .{ }^{14}$ In Fig. 3(a), we plot the maximum cooling power $\dot{Q}_{\max }$ as a function of temperature $T$. As in the ballistic junction limit $\dot{Q}_{\max }$ increases dramatically by increasing $P$ reaching much larger values than for a diffusive N/I/S cooler [the dotted line in Figs. 3(a) and 3(b)], which is achieved in the case of $\mathcal{A}=1 \mu \mathrm{m}^{2}, \quad \sigma_{N}=0.015(\mu \Omega \mathrm{cm})^{-1}, \quad R_{N}$ $=2.7 \mathrm{M} \Omega$, and $T / T_{c}=0.45$. Moreover, the window of positive values for $\dot{Q}_{\max }$ is larger for the larger $P$. As for the N/I/S junctions, there is a maximum value of temperature, $T_{\max }$ $\sim 0.75 T_{c}$ for which cooling is achieved [cf., Fig. 3(a)]. This maximum value holds for a wide range of parameters ${ }^{10,12}$ and is due to the increase of the number of thermally excited quasiparticles that contribute to the Joule heat. By lowering the temperature from $T=T_{\max }$, the cooling power (at optimal bias $V_{\text {opt }}$ ) first increases, reaches a maximum, and finally decreases due to the Joule heat produced by the Andreev processes. At certain temperature the cooling power tends to zero, which defines the lowest temperature for the cooling regime.

In Fig. 3(b) we show the dependence of the cooling power on the spin-filter efficiency $P$. For all temperatures 
$\dot{Q}_{\max }$ increases monotonically by increasing $P$. The efficiency of a refrigerator is characterized by the ratio between the optimum cooling-power and total input power: $\eta=\dot{Q}_{\max } / I V_{\text {opt }}$. The inset of Fig. 3(b) shows the temperature $T$ dependence of $\eta$ for several $P$ values. For a fully polarized SF barrier $(P=1), \eta$ reaches up to $23 \%$, which is mach larger than that for the optimum N/I/S cooler $(\eta=15 \%)$ and comparable to that for a half-metallic N/FM/S cooler with $P=1{ }^{3}$ We can then conclude that the spin-filtering effect gives rise to highly efficient refrigeration.

Next we evaluate the final electron temperature of the normal metal, so we need to consider the mechanism of energy transfer. In this case the electron temperature depends on the efficiency with which heat generated in the electron population can be transferred to the low-temperature bath. Due to the voltage biasing some power is dissipated as heat, this power is supplied initially to the electrons in the metal and is transmitted to the bath by phonons. In thin films at low temperatures, the wavelength of a thermal phonon is much less than the film thickness so the temperature of the phonons is the same as the temperature of the bath, the lattice temperature $T_{l}$. Then the electron temperature is determined by the rate at which electrons can transfer energy to the phonons which is given by $\dot{Q}_{e-l}=\Sigma \mathcal{V}\left(T_{e}^{5}-T_{l}^{5}\right)$, where $\mathcal{V}$ is the volume of $\mathrm{N}, T_{e(l)}$ is the electron (lattice) temperature, and $\Sigma$ is a material dependent parameter. ${ }^{3}$ The final electron temperature $T_{e}$ is determined by the energy-balance equation $\dot{Q}\left(T_{e}, T_{l}\right)+\dot{Q}_{e-l}\left(T_{e}, T_{l}\right)=0$, where we set the temperature of the superconductor $\left(T_{S}\right)$ equal to the bath temperature $T_{l}$. In order to understand the behavior of the final electron temperature, Fig. 3(c) shows $T_{e}$ as a function of bias voltage for 4 different $P$ in the case of the starting lattice temperature $T_{l}=300 \mathrm{mK}$. The junction parameters were taken according to experimental values: ${ }^{3,11} \Sigma=2 \times 10^{-9} \mathrm{WK}^{-5} \mu \mathrm{m}^{-3}, \mathcal{V}$ $=0.5 \mu \mathrm{m}^{3}$ and assuming that $\mathrm{Al}$ is the superconductor $(\Delta=180 \mu \mathrm{eV})$. Fig. 3(c) shows a remarkable reduction of $T_{e}$, as we increase the value of voltage, the temperature tends to lower until it reaches a optimum voltage $\left(e V \sim \Delta_{0}\right)$. We observe that the increment of $P$ reduces drastically the minimum electron temperature, i.e., $T_{e}^{\mathrm{min}} \sim 50 \mathrm{mK}$ for $P=1$. In the inset of Fig. 3(c) we plot the minimum electron temperature $T_{e}^{\mathrm{min}}$ vs the starting lattice temperature $T_{l}$. The straight dotted line marked $T_{e}^{\min }=T_{l}$ as a reference. The result indicates that in a wide $T$ range, we can effectively cool down the electron temperature of $\mathrm{N}$.

In summary, we have proposed an electron-refrigerator based on spin-filter barriers. Due to the suppression of the Andreev Joule heating N/SF/S junctions can achieve values for the cooling power higher than those predicted for conventional N///S coolers. Refrigeration efficiency of $15 \%-23 \%$ can be achieved by using well known spin-filters barriers, for example, EuS/Al $(P \sim 0.86),{ }^{14} \mathrm{EuSe} / \mathrm{Al}(P \sim 1),{ }^{21}$ and GdN/ $\mathrm{NbN}$ junction $(P \sim 0.75){ }^{22}$ We also expect a similar effect using spinel ferrites (e.g., $\left.\mathrm{NiFe}_{2} \mathrm{O}_{4}\right)^{23,24}$ or manganites (e.g., $\mathrm{LaMnO}_{3+\delta}$ and $\left.\mathrm{Pr}_{0.8} \mathrm{Ca}_{0.2} \mathrm{Mn}_{1-y} \mathrm{Co}_{y} \mathrm{O}_{3}\right)^{25,26}$ as spinfilters. Our results open up a way to make efficient solidbased refrigerators for the cooling of practical devices, like superconducting X-ray detectors, single-photon detectors, magnetic sensors, nano-electromechanical systems, and qubits.

We would like to thank S. Kashiwaya, M. Koyanagi, and S. Nakamura for useful discussions and comments. This work was supported by the Topological Quantum Phenomena (No. 22103002) KAKENHI on Innovative Areas, the JSPS Institutional Program for Young Researcher Overseas Visits, the European Union Seventh Framework Programme (FP7/ 2007-2013) under grant agreement "INFERNOS" No. 308850, the Spanish Ministry of Economy and Competitiveness under Project FIS2011-28851-C02-02, the CSIC, and the European Social Fund under JAE-Predoc program.

${ }^{1}$ M. Nahum, T. M. Eiles, and J. M. Martinis, Appl. Phys. Lett. 65, 3123 (1994).

${ }^{2}$ M. M. Leivo, J. P. Pekola, and D. V. Averin, Appl. Phys. Lett. 68, 1996 (1996).

${ }^{3}$ F. Giazotto, T. T. Heikkilä, A. Luukanen, A. M. Savin, and J. P. Pekola, Rev. Mod. Phys. 78, 217 (2006).

${ }^{4}$ J. T. Muhonen, M. Meschke, and J. P. Pekola, Rep. Prog. Phys. 75, 046501 (2012).

${ }^{5}$ A. M. Clark, N. A. Miller, A. Williams, S. T. Ruggiero, G. C. Hilton, L. R. Vale, J. A. Beall, K. D. Irwin, and J. N. Ullom, Appl. Phys. Lett. 86, 173508 (2005).

${ }^{6}$ G. C. O’Neil, P. J. Lowell, J. M. Underwood, and J. N. Ullom, Phys. Rev. B 85, 134504 (2012).

${ }^{7}$ P. J. Lowell, G. C. O’Neil, J. M. Underwood, and J. N. Ullom, Appl. Phys. Lett. 102, 082601 (2013).

${ }^{8}$ A. Bardas and D. Averin, Phys. Rev. B 52, 12873 (1995).

${ }^{9}$ S. Rajauria, P. Gandit, T. Fournier, F. W. J. Hekking, B. Pannetier, and H. Courtois, Phys. Rev. Lett. 100, 207002 (2008).

${ }^{10}$ A. S. Vasenko, E. V. Bezuglyi, H. Courtois, and F. W. J. Hekking, Phys. Rev. B 81, 094513 (2010).

${ }^{11}$ F. Giazotto, F. Taddei, R. Fazio, and F. Beltram, Appl. Phys. Lett. 80, 3784 (2002).

${ }^{12}$ A. Ozaeta, A. S. Vasenko, F. W. J. Hekking, and F. S. Bergeret, Phys. Rev. B 85, 174518 (2012).

${ }^{13}$ S. A. Wolf, D. D. Awschalom, R. A. Buhrman, J. M. Daughton, S. von Molnár, M. L. Roukes, A. Y. Chtchelkanova, and D. M. Treger, Science 294, 1488 (2001).

${ }^{14}$ J. S. Moodera, T. S. Santos, and T. Nagahama, J. Phys.: Condens. Matter 19, 165202 (2007).

${ }^{15}$ S. Kashiwaya, Y. Tanaka, N. Yoshida, and M. R. Beasley, Phys. Rev. B 60, 3572 (1999).

${ }^{16}$ F. S. Bergeret, A. Verso, and A. F. Volkov, Phys. Rev. B 86, 214516 (2012).

${ }^{17}$ S. Kawabata, S. Kashiwaya, Y. Asano, Y. Tanaka, and A. A. Golubov, Phys. Rev. B 74, 180502(R) (2006).

${ }^{18}$ G. E. Blonder, M. Tinkham, and T. M. Klapwijk, Phys. Rev. B 25, 4515 (1982).

${ }^{19}$ W. Belzig, F. K. Wilhelm, C. Bruder, G. Schön, and A. D. Zaikin, Superlattice Microstruct. 25, 1251 (1999).

${ }^{20}$ K. D. Usadel, Phys. Rev. Lett. 25, 507 (1970).

${ }^{21}$ J. S. Moodera, R. Meservey, and X. Hao, Phys. Rev. Lett. 70, 853 (1993).

${ }^{22}$ K. Senapati, M. G. Blamire, and Z. H. Barber, Nat. Mater. 10, 849 (2011).

${ }^{23}$ U. Lüders, M. Bibes, K. Bouzehouane, E. Jacquet, J.-P. Contour, S. Fusil, J.-F. Bobo, J. Fontcuberta, A. Barthélémy, and A. Fert, Appl. Phys. Lett. 88, 082505 (2006).

${ }^{24}$ Y. K. Takahashi, S. Kasai, T. Furubayashi, S. Mitani, K. Inomata, and K. Hono, Appl. Phys. Lett. 96, 072512 (2010).

${ }^{25}$ D. K. Satapathy, M. A. Uribe-Laverde, I. Marozau, V. K. Malik, S. Das, Th. Wagner, C. Marcelot, J. Stahn, S. Brück, A. Rühm, S. Macke, T. Tietze, E. Goering, A. Franó, J.-H. Kim, M. Wu, E. Benckiser, B. Keimer, A. Devishvili, B. P. Toperverg, M. Merz, P. Nagel, S. Schuppler, and C. Bernhard, Phys. Rev. Lett. 108, 197201 (2012).

${ }^{26}$ T. Harada, I. Ohkubo, M. Lippmaa, Y. Sakurai, Y. Matsumoto, S. Muto, H. Koinuma, and M. Oshima, Phys. Rev. Lett. 109, 076602 (2012). 